\title{
A Latent Measure of Political Protest*
}

\author{
Erica Chenoweth ${ }^{\dagger}$ Vito D’Orazio; and Joseph Wright ${ }^{\S}$
}

March 25, 2014

Prepared for the Annual Meeting of the International Studies Association

March 29, 2014 (Toronto, ON)

\begin{abstract}
In recent years, scholars have developed a number of new databases with which to measure protest. Although these databases have distinct coding rules, all attempt to capture incidents of social conflict. We argue, however, that due to a variety of sources of measurement error, subjective coding decisions, and operational specifications, no single indicator of protest adequately measures how much protest exists in a given place at a given time. As a result, empirical studies that employ these measures yield inferences with limited generalizability. To increase the generalizability of the empirical findings, we suggest using an Item Response Theory (IRT) approach to estimate a latent dimension of protest using nine different protest measures that vary in their operational specifiations as well as their temporal and spatial coverage. The estimates of the IRT models are used in two ways. First, to demonstrate how existing measures differ, the IRT's item estimates are used to compare the nine measures of protest based on their degree of diffculty (the quantity of latent protest required to observe a ' 1 ' in the data) and their ability to discriminate (the speed with which changes in the latent quantity of protest affect the probability of observing a ' 1 ' in the data). Second, the estimated quantity of protest is applied to both monthly and yearly models of authoritarian breakdown. The results demonstrate that the latent protest variable increases the out-of-sample classification of authoritarian breakdown events; and improves in-sample prediction relative to existing global protest variables. Our study illustrates the potential value of modeling a latent dimension of protest rather than solely relying on observed indicators.
\end{abstract}

\footnotetext{
*The author order is alphabetical. This research was conducted for the Political Instability Task Force (PITF). The PITF is funded by the Central Intelligence Agency (CIA). The views expressed herein are the authors' alone and do not necessarily represent the views of the Task Force or the U.S. Government.

${ }^{\dagger}$ Email: Erica.Chenoweth@du.edu. Corresponding author.

${ }^{\ddagger}$ Email: dorazio@iq.harvard.edu

$\S$ Email: josephgwright@gmail.com
} 
When protests began in Ukraine in late 2013, many were wondering what effect they would have on outcomes such as the durability of the regime, regional power relations, and future international influences on the country. A common response to these questions is that it depends on how much protest we observe. Indeed, in recent years, scholars have developed a number of new databases with which to measure protest. ${ }^{1}$ Although these databases have distinct coding rules, all attempt to capture incidents of social conflict. Yet, reliably observing the quantity of protest in a country presents numerous challenges. We argue that due to a variety of sources of measurement error due to media coverage, subjective coding decisions, and operational specifications, no single indicator of protest adequately measures how much protest exists in a given place at a given time. Those that do measure some quantity of protest, such as the Social Conflict in Africa Database (SCAD), are often quite limited in terms of spatial or temporal coverage (Ukraine, for example, is beyond the scope of SCAD). Moreover, operational definitions of protest, source materials, and coding decisions may vary widely across protest measures. As a result, empirical studies that employ these measures yield inferences based on the operationalization of an individual measure rather than inferences based on the degree of protest.

In many ways, the issue here is similar to measurement of many different types of political phenomena, including democracy (Treier and Jackman 2008; Pemstein, Meserve and Melton 2010) and corruption (Pieroni, d'Agostino and Bartolucci 2013). In all such cases, the indicators commonly used to represent different classifications of political phenomena - or different political events - are not observed directly, and are simply operationalized using theoretical assumptions (implicitly or explicitly). In making these conceptual distinctions, researchers may introduce measurement error into the operationalization of these concepts in ways that influence the research findings scholars produce when they use these indicators.

In an effort to improve the measurement of protest, we combine nine different protest data sets in an Item Response Theory (IRT) framework to estimate a latent measure reflecting the quantity of protest at two levels of analysis (country-year and country-month). This is a useful approach because each dataset relies on different sources - including, in the case of the Major Episodes of Contention Data Project (MEC), news sources supplemented with historical research - such that cross-validation and inputs of missing information may improve the data's coverage. The IRT framework also provides a set of item parameters that describe the relationship of these variables to one another. For example, the analysis of these parameters shows SCAD to be a liberal measure of protest, while MEC is consistently shown as a conservative measure. Banks' protest measure, the most common in the literature, is somewhere in the middle, but contributes more information to the underlying latent measure than others.

The IRT models estimate a latent trait for each observation (a quantity of observed protest) that is explored by applying it to a model of authoritarian breakdown. The results demonstrate that the latent protest variable increases the out-of-sample classification of authoritarian breakdown events; and improves in-sample prediction relative to existing global protest variables. Our study illustrates the value of modeling a latent variable rather than solely relying on observed indicators of protest.

\footnotetext{
${ }^{1}$ See Appendix for a full discussion of the protest variables.
} 


\section{Weaknesses of Observed Protest Indicators}

Individual attempts to measure a quantity of social protest may be inadequate for three reasons: measurement error due to media coverage, subjective coding decisions, and varying operational specifications. Even in cases where efforts are made to avoid measurement error, minimize subjectivity, and generalize the concept to a degree where various operational specifications can be obtained using the same data, there are often limits to the scope of coverage. As a result, the inferences we draw from empirical models are tied to the idiosyncrasies of the protest measure, with varying generalizability to the latent concept of interest.

There are three primary sources of measurement error due to media coverage. First, scholars have long noted a tendency for the news media to under-report nonviolent events, like protests, compared with violent ones, like bombings or attacks (Smith et al. 2001). Because news sources (e.g. newspapers and newswires) are the most common source from which protest researchers develop these databases, such datasets may not represent the full universe of events - a problem that is particularly acute in protest data sets that rely on a single news source, such as the New York Times. Such data sets quite literally only capture protests "observed by a New York Times reporter," which limits the reliability of such indicators when considered in isolation from other news reports.

A related problem is that the systematic under-reporting of protest events in single news sources is probably even more pronounced than for violent incidents, since information on nonviolent action is arguably much easier for governments to suppress. It is likely easier to deny a protest by unarmed persons than an armed attack, particularly when the protest is relatively small or subtle. For instance, Herkenrath and Knoll (2011) find that protest databases that rely solely on international media sources - as do Banks and SCAD - systematically under-report protest activity in some countries, where such coverage is generally lacking due to disinterest or censorship.

Third, many forms of protest - such as everyday forms of resistance, certain symbolic protests, and stay-at-home demonstrations - are unlikely to obtain meaningful news coverage, at least on an incident-level basis (Scott 1985). This means that news coverage tends to converge on large, spectacular, and visually-stimulating forms of protest while ignoring more mundane, day-to-day forms. Yet the latter can often be highly influential in developing the capacity and "cognitive liberation" for a larger social movement to emerge (McAdam 1999). Moreover, this bias renders inaccurate claims that existing protest databases capture all protest incidents; we know, or at least we suspect, that they systematically undercount contentious activity in other forms. Because of these problems, some data sets rely on both international and local news reports (e.g. Regan), or they supplement news reports with additional historical research (e.g. MEC). This provides an opportunity to leverage information from data sets relying on different source material to compensate for potential measurement error through under-reporting.

Subjective coding decisions represent another mechanism by which individual measures of

protest may be inadequate for measuring the degree of protest. For example, existing datasets may draw on different primary and secondary sources in an attempt to mitigate the effects of systematic media bias. Source selection is subjective, however, and ultimately this decision may induce any or all of the three types of measurement error described above.

More generally, subjective coding decisions are made whenever the operational definition lacks the ability to clearly delineate an observation. Regardless of how precise the operationalization is, subjectivity creeps into the data in this way. In such instances, the decision to include the observation, or to assign a score to that observation, is a function of the principal investigator or 
research assistant more than anything else. Using a variety of measures may help yield consensus on such observations, even if the estimated latent quantity has larger confidence intervals than observations for which there is more consistency.

A third issue when measuring protest is that operational specifications differ from measure to measure. While this feature is certainly not unique to protests, it appears to be exceedingly problematic in this literature. For example, a SCAD protest can consist of fewer than 10 individuals while an MEC protest requires a minimum of 1,000. While such definitions are typically clear, it is difficult to quantitatively assess the effect of a protest when the potential differences in operationalization are so great.

Returning to Ukraine, consider a scenario wherein a researcher turned to existing models for evidence about whether protests would lead to removal of the incumbent leader. Perhaps one model that utilizes a liberal measure of protest, such as the Armed Conflict Location and Event Dataset (ACLED), predicts no removal of the incumbent, while another model that utilizes a conservative measure, such as MEC, predicts the incumbent's removal. In such cases, the different operational specifications are a source of confusion. A superior approach may be a model that estimates a latent quantity of protest, and uses that quantity in the forecasting model. In this way, analysts can state, for example, that x-unit increases along this latent dimension correspond to y-unit increases in the likelihood of an event. To position the Ukraine protest on the latent dimension, the analyst may assess the degree of protest as compared to other protests whose latent quantity has been estimated, and make well-informed forecasts as to the outcome of the event.

Measurement error, in the form of systematic under-reporting, information suppression, and the diversity of nonviolent actions may yield disagreement across different protest datasets. So might subjective coding decisions and various operational specifications. The validation problems created by these issues becomes apparent when we compare data sets with seemingly comparable conceptual definitions. If high amounts of measurement error were present, we would see very little correspondence across datasets in incidents of reported protest. Consider Figure 1, which shows annual observations of seven protest databases focused on a single country (Tunisia). ${ }^{2}$ The table illustrates how even within a single country, the correspondence and convergence of protest observations across datasets is actually quite scarce. Indeed, the only year in which all available datasets reported protests in Tunisia was in 2011 - that is a single year in a 32-year time series (just $3 \%$ of the observations).

To construct a generalizable measure that reflects the degree of protest a country is experiencing, existing measures are combined to reflect a single, latent dimension of social protest. The latent dimension is a measure of the quantity of social protest that a state is experiencing in a particular year (or month). The method used for estimating the latent trait is item response theory (IRT).

IRT has its roots in applications to standardized testing, whereat is widely used because it does not require strict, a priori rules for combining test questions into a score (Linden and Hambleton 1997). Rather, in the IRT framework it is assumed that each individual test-taker possesses some quantity of a latent trait, commonly referred to as ability, and the individual's responses to test questions are a function of their latent ability. In other words, each test-taker's response on an exam is an observable indicator that provides some information that helps to assess the test-taker's ability. The impact of a particular response on an individual's score is a function of the question asked and how it compares to other questions asked, who and how many other test-takers answered that question correctly, and what other questions those individuals answered correctly.

\footnotetext{
${ }^{2}$ LAPP and EPCD are omitted due to lack of geographic coverage.
} 


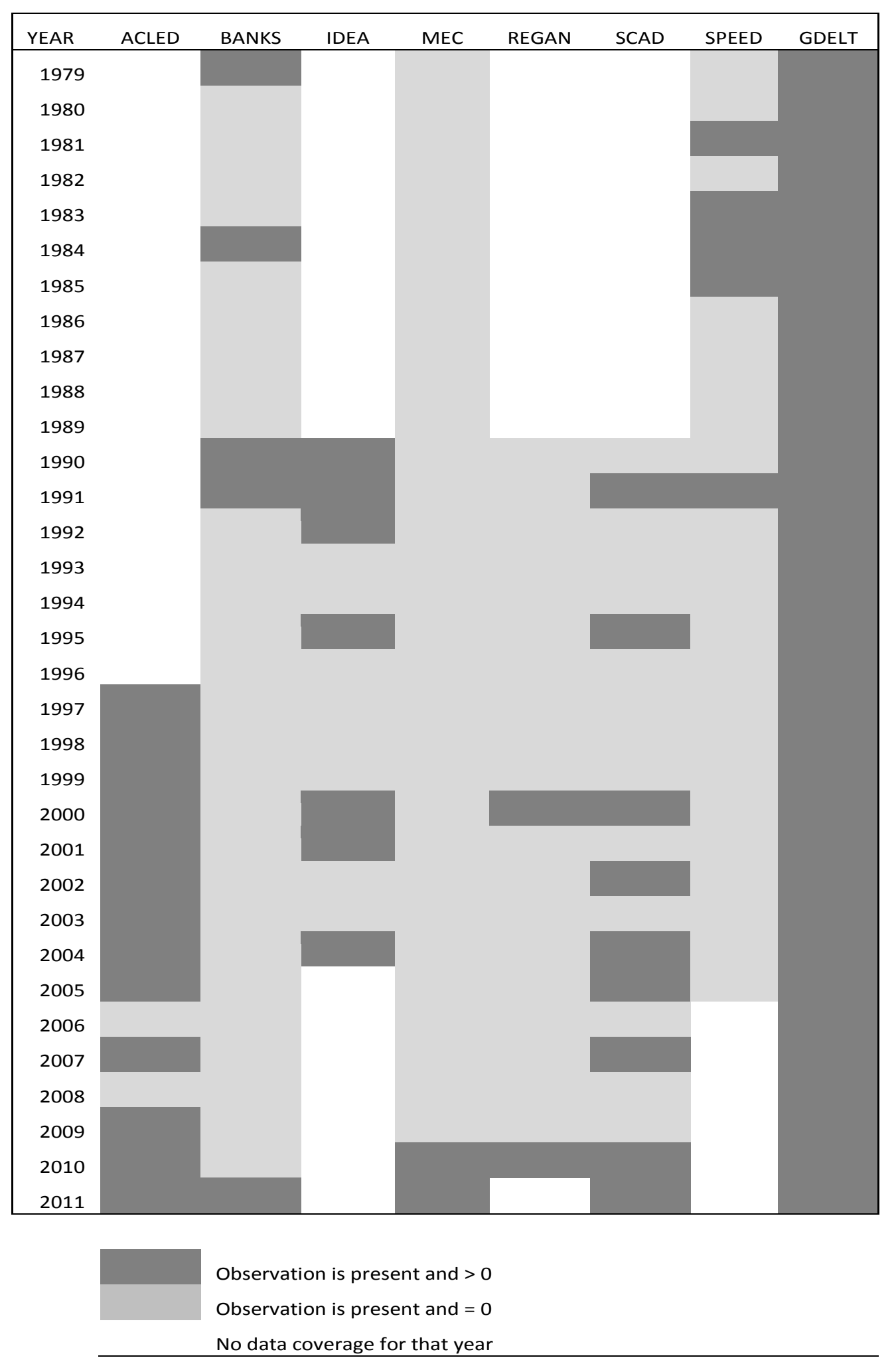

Figure 1: Annual Observations of Eight Protest Datasets in Tunisia, 1979-2011. See Appendix for information on the protest datasets. 
In the present application, the items correspond to the nine measures of social protest, and the unit of analysis is the country-year (or country-month), as opposed to the individual test-taker. Each country-year is assumed to have some latent quantity of protest that manifests itself in the observed response patterns contained in the nine protest measures. Those country-years where a protest is observed in all relevant measures are assumed to have the largest quantity of protest, while those country-years where no protest is observed in any measure are assumed to have the smallest quantity of protest.

In Political Science, IRT models have been used, for example, to study regime types (Treier and Jackman 2008; Pemstein, Meserve and Melton 2010), military and security relationships (Benson and Clinton 2012; D'Orazio 2013), and political ideology (Clinton and Jackman 2009). In each of these applications, observable indicators of the latent concept are used to estimate a continuous, latent trait. For example, Treier and Jackman (2008) estimate regime types, a latent concept, using the Polity project's categorical indicators, which are the components of the commonly used 21-point Polity Scale (Marshall and Jaggers 2007).

The present application is similar, although not identical. We have multiple measures of the same concept, rather than the component indicators of a single measure. By using the same component indicators, Treier and Jackman (2008) uphold Polity's view on which components are necessary to measure the concept of regime type. Their argument pertains to how the indicators are combined to reflect how democratic a state is. Here, we do not maintain that there is a single definition of the concept of a social protest, but that numerous operationalizations exist and the various measures reflect different coding decisions and stress different attributes of protest. Similar to how higher scores reveal a state's level of democracy for Treier and Jackman (2008), higher scores reflect the notion that a state is experiencing a larger quantity of protest.

The contribution of each protest measure to the estimated quantity of protest depends, in part, on how these measures relate to one another. Specifically, the IRT models applied here estimate parameters that describe each measure's degree of difficulty - the quantity of latent protest required to observe a ' 1 ' in the data - and their ability to discriminate - the speed with which changes in the latent quantity of protest affect the probability of observing a ' 1 ' in the data. These parameters quantify similarities and differences of existing measures and, if differences are observed, help to justify the application of IRT models to the measurement of social protest.

Indeed, differences among the protest measures are observed. MEC is a consistently conservative coding scheme, while ECPD, SCAD, and ACLED are quite liberal. SPEED is surprisingly conservative, given its event-based nature, and perhaps reflects the extensive information processing that the SPEED project undertakes. Banks is somewhere in the middle, but contributes the most information to the estimation of the latent quantity of protest. In the monthly models, Banks is dropped due to the lack of data, and SPEED and ACLED provide the most information. Finally, SCAD contributes very little to the count models, demonstrating its operational definition is not consistent with other measures.

\section{Protest Data and IRT Models}

IRT models have been discussed in a number of existing studies (Baker 2001; Baker and Kim 2004; Samejima 1997; Reise and Waller 2009; Toland 2013). This section briefly introduces two IRT models that are appropriate for the protest data: the two parameter logistic (2PL) and the graded 
response model (GRM). ${ }^{3}$ While the $2 \mathrm{PL}$ and GRM are estimated under a number of different specifications, as shown in Table 1, the models explored in this section are the yearly-level binary and count models for the full temporal coverage. ${ }^{4}$

\begin{tabular}{lcll}
\hline Spatial & Temporal & Aggregation & Response \\
\hline Post CW & $1990-2012$ & Monthly & Binary \\
Post CW & $1990-2012$ & Monthly & Count \\
Post CW & $1990-2012$ & Yearly & Binary \\
Post CW & $1990-2012$ & Yearly & Count \\
All & $1946-2012$ & Monthly & Binary \\
All & $1951-2012$ & Monthly & Count \\
All & $1946-2012$ & Yearly & Binary \\
All & $1951-2012$ & Yearly & Count \\
\hline
\end{tabular}

Table 1: Model Information

The type of response, or the level at which the protest is measured, dictates the set of IRT models that may be applied. A binary response refers to the case where the protest measures are simply coded for the presence of a protest in a given country-year. A more accurate measure might be based on the count of the number of protests in a given country-year. For such models, the count data are reduced to the ordinal level. ${ }^{5}$ To construct these ordinal variables, each count is reduced to at most nine categories plus an 'NA' category by splitting off the observed zeros, which becomes Category 1, and then ordering the data and splitting it into eight bins, some of which are empty. The empty bins are removed, with the result being an ordered variable that represent categories constructed from the initial protest count.

As an example of what the ordinal data look like, Table 2 shows the cross tabulation for "All Year Count." Each row sums to 11,780 - the number of observations in the yearly count data. ECPD, LAPP, and SCAD are represented in all nine categories, and ACLED, MEC, and Regan in eight categories. SPEED and Banks reduce to six, the fewest number of categories for any of the indicators. As can be seen in the NA column, there is missingness in all the indicators, with MEC having the least and LAPP having the most. However, this is an ignorable missing data problem, as the missingness is not related to $\theta$, but to the scope of the original data collection project (?).

\section{The 2PL Model}

The 2PL is an appropriate IRT model for binary response indicators, which is a common variable type in the social protest literature. For example, Banks is the most commonly used measure of

${ }^{3}$ The IRT models are estimated using the mirt package in R (Version mirt_1.1 (Chalmers 2012) and R 3.0.2 GUI 1.62 Snow Leopard build (6558)). For additional information about the model and parameter estimation, see Chalmers (2012).

${ }^{4}$ For the binary data, the models' timespan is 1946 through 2012, and for the count data its 1951 through 2012. Models have also been estimated at the monthly level and for the post-Cold War period. As a robustness check, each has also been estimated as a two-dimensional model.

${ }^{5}$ The GRM assumes ordinal item responses. Although more flexible methods for estimating latent traits exist (?), reducing the responses to ordinal categories appears to reasonably reflect degrees of social protest. 
Table 2: Reduction for "All Year Count"

\begin{tabular}{|l|r|r|r|r|r|r|r|r|r|r|}
\hline & 1 & 2 & 3 & 4 & 5 & 6 & 7 & 8 & 9 & NA \\
acled & 272 & 209 & 46 & 46 & 69 & 65 & 70 & 73 & & 10,930 \\
banks & 4801 & 1593 & 419 & 258 & 378 & 308 & & & & 4,023 \\
ecpd & 48 & 52 & 49 & 50 & 50 & 50 & 50 & 50 & 50 & 11,332 \\
idea & 1515 & 609 & 147 & 91 & 175 & 146 & 167 & & & 8,930 \\
lapp & 17 & 6 & 3 & 4 & 4 & 4 & 4 & 4 & 4 & 11,730 \\
mec & 10577 & 56 & 56 & 56 & 57 & 156 & 54 & 12 & & 756 \\
regan & 2036 & 231 & 112 & 85 & 86 & 90 & 99 & 99 & & 8,942 \\
scad & 344 & 116 & 86 & 90 & 100 & 95 & 122 & 72 & 97 & 10,658 \\
speed & 8467 & 1202 & 205 & 138 & 225 & 213 & & & & 1,330 \\
\hline
\end{tabular}

protest, and it is commonly used at the binary level. The "two parameter" portion of $2 \mathrm{PL}$ refers to the discrimination and difficulty parameters, the IRT estimates that tell us how these protest measures relate to one another, given the estimated latent quantity of protest.

Let $\theta$ be the latent trait, $i=1, \ldots, N$ be the country-years in the data, and $j=1, \ldots, n$ be the measures of protest (e.g., ACLED or Banks). Let the item discrimination parameter be $\alpha_{j}$, and the item difficulty parameter be $d_{j}$. $D$ is a scalar for consistency of interpretation, and is set to approximately $1.702 .{ }^{6}$ The probability of a country-year receiving a score of ' 1 ' for a given protest measure is shown in Equation 1.

$$
P\left(x_{i j} \mid \theta_{i}\right)=\frac{1}{1+\exp \left[-D \alpha\left(\theta_{i}-d\right)\right]}
$$

Equation 1 is the foundation of IRT models and is known as the item response function (IRF). IRFs are estimated for each item, as shown in Figure 2 for the "All Year Binary" model. On the $\mathrm{x}$-axis is $\theta$, or the estimated quantity of protest, and on the $\mathrm{y}$-axis is the probability of a observing a positive response. Each IRF is described by its discrimination and difficulty parameters. The discrimination parameter is an estimate of how well an item can distinguish between values of $\theta$. The IRF of a well-performing indicator has a steep slope, while an item with no ability to discriminate among individuals at various levels of $\theta$ will appear flat. In other words, a flat IRF suggests that the probability of observing a ' 1 ' in the data is always the same, regardless of the latent quantity of protest. The item difficulty is the point at which the probability of observing a positive response (' 1 ' in the data) is equal to the probability of observing a negative response (' 0 ' in the data). For items with a high degree of difficulty, the IRFs appear shifted to the right, suggesting a protest is only observed for those country-years with large quantities of protest. At the other end, a low degree of difficulty is represented by IRFs shifted to the left. For such indicators, only a small quantity of protest is necessary to observe a positive response.

What is desirable from the IRFs in this application of a $2 \mathrm{PL}$ model are steep curves scattered across the range of $\theta$. This would suggest that each measure of protest distinguishes well, and that enough measures are included to have confidence in the estimates across the range of $\theta$. To some degree, this is what is observed in Figure 2.

\footnotetext{
${ }^{6}$ The notation is consistent with Chalmers (2012).
} 


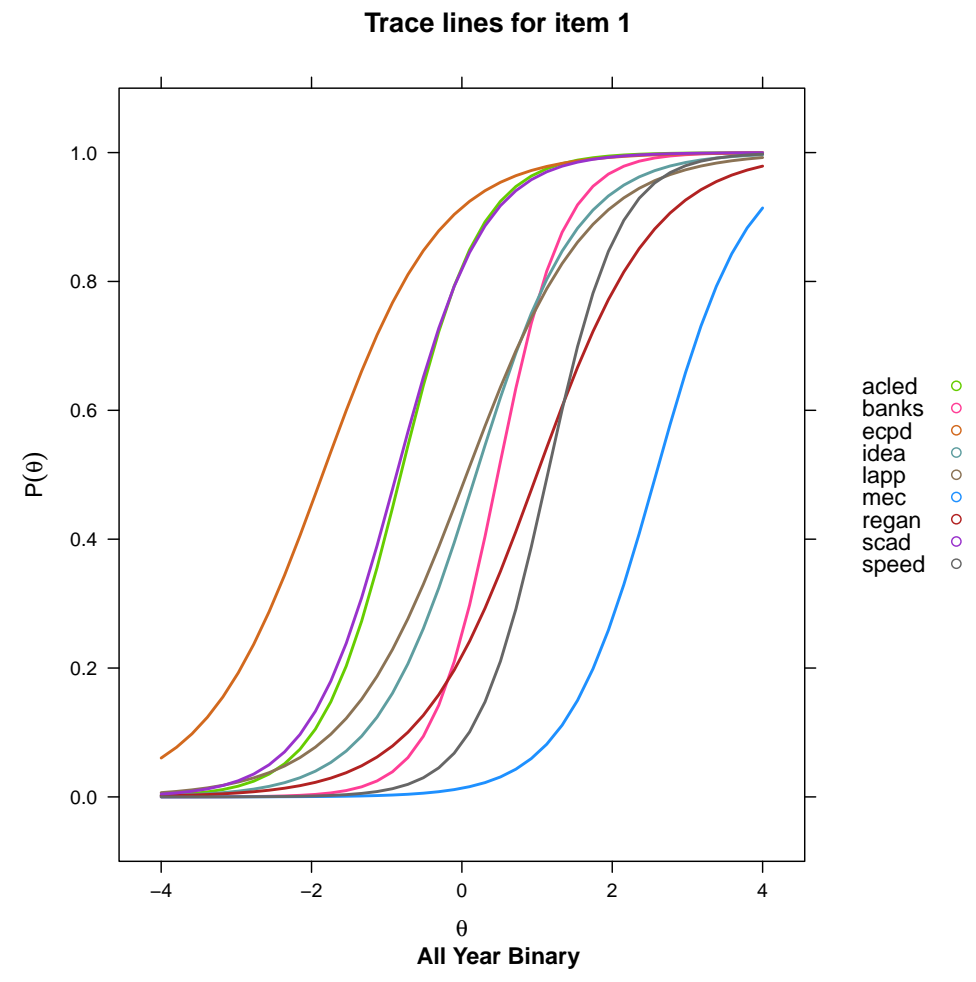

Figure 2: IRF: All Year Binary

In Figure 2, LAPP is the flattest indicator, followed by Regan and ECPD. However, they are not alarmingly flat to warrant dropping them from the model. ${ }^{7}$ Banks and SPEED are the steepest, suggesting these protest measures distinguish among values of $\theta$ more strongly than others. In general, however, the primary difference in these protest measures is not their discrimination, but their difficulty.

The MEC measure has the highest degree of difficulty, as shown by its location, or shift to the right. This suggests that a positive response in the MEC data is increasingly likely in states where large quantities of protest are observed. In comparison, ECPD is shifted to the left, suggesting that only a small amount of protest is necessary for the ECPD to be likely to record a positive response. SCAD and ACLED have lower degrees of difficulty as well, which is not surprising given their event-based approach. By contrast SPEED, which is also an event-based dataset, has a considerably high estimate of difficulty. This may reflect the extensive information processing that the SPEED project undertakes, or perhaps it signals a more conservative operational definition of protest.

From the IRT estimates, we calculate a set of item information functions (IIF) to examine the information contributed to $\theta$ by each protest measure. Ideally, one would want to see a high degree of certainty across a wide range of $\theta$, demonstrating confidence in the full spectrum of point estimates. The more information contributed, the greater the certainty that has been ascertained

\footnotetext{
${ }^{7}$ In prior models, measures of protest derived from Global Data on Events, Location, and Tone (GDELT) were included. These IRFs were incredibly flat, and were ultimately rejected as valid measures of protest.
} 
for that particular value of $\theta$. Overlapping IIFs signal commonalities in the quantity of protest at which the items contribute information, and some take this as evidence to drop one of the indicators (Toland 2013). Here, indicators are not dropped, but rather this is taken as evidence that such measures are similar, perhaps reflecting a common operational definition. The formula for calculating the IIF is $I_{i}(\theta)=\alpha_{i}^{2} P_{i}(\theta) Q_{i}(\theta)$, where $Q_{i}(\theta)=1-P_{i}(\theta)$.

\section{Item information trace lines}

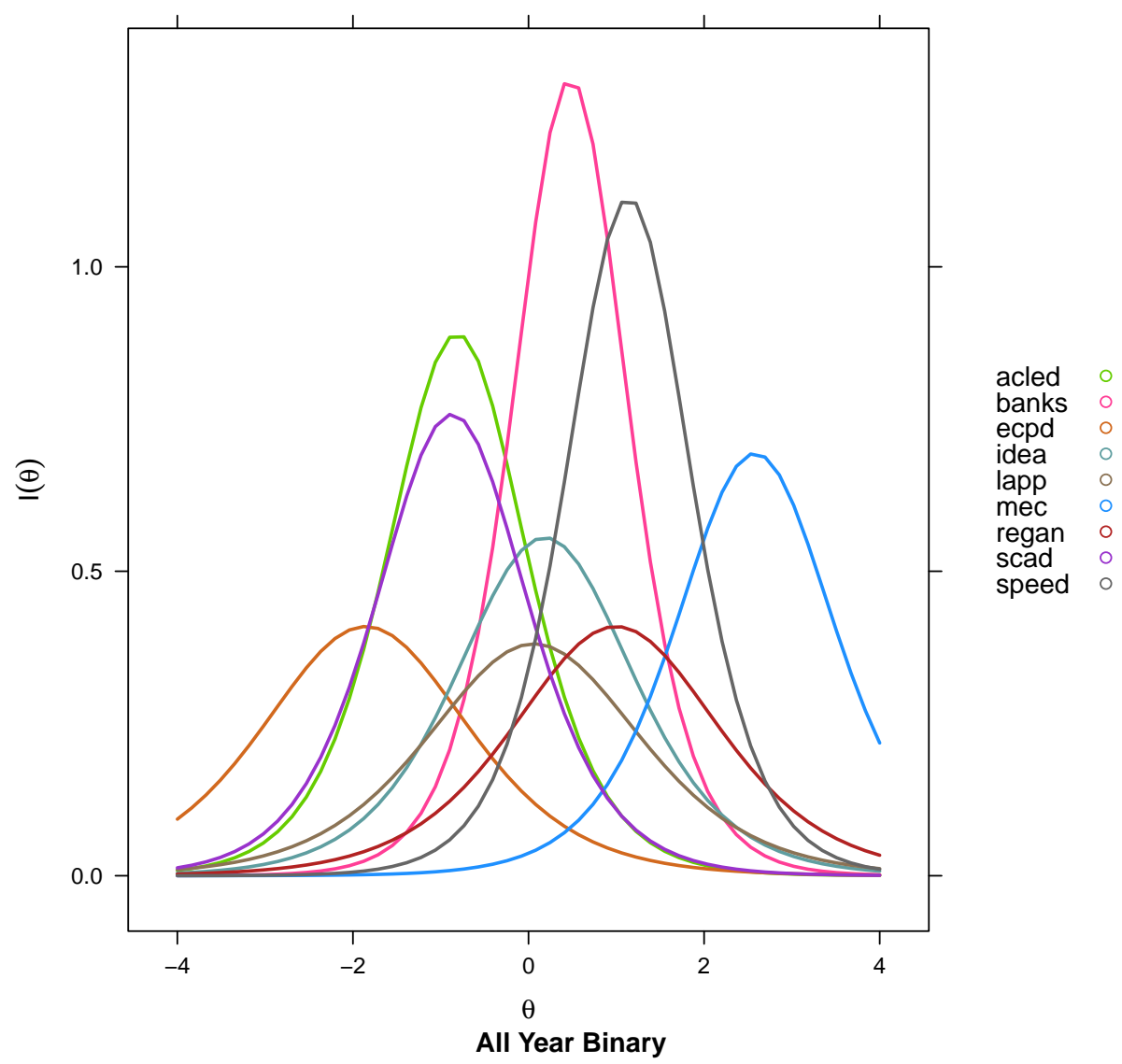

Figure 3: IIF: All Yearly Binary

The IIFs from the "All Yearly Binary" model are plotted in Figure 3. The IIF whose contribution is at the lowest values of $\theta$, is ECPD, while the IIF whose contribution is for the highest values of $\theta$ is for MEC. Notice, the location of the highest point in each IIF corresponds to the item's difficulty parameter. LAPP and IDEA contribute around $\theta$ values of 0 , and are similar to Banks but with considerably less information. The same applies to Regan, but at a $\theta$ value similar to SPEED. Notice, the amount of information, or the height of the IIF, corresponds to the item's discrimination parameter.

Figure 3 also shows two sets of two IIFs with a high degree of information and quite a large overlap: Banks and SPEED, and ACLED and SCAD. This suggests that these datasets contain comparable information on social protest, and may be interchangeable with each other in statistical analyses. The strongest similarity among indicators is seen for ACLED and SCAD, which are shown 
to contribute for lower values of $\theta$, consistent with their event-based coding schemes. SPEED, however, is also an event-level dataset, and yet its impact on $\theta$ is primarily at the higher levels. SPEED and Regan are quite similar as well, albeit with much more information contributed by SPEED.

The model estimates for the "All Year Binary" model are in Table 3. The discrimination parameter estimates range from LAPP's 1.28 to Banks' 2.288, and there is considerable variation another the remaining protest measures within that range. As per item difficulty, MEC is shown to be the most difficult, at -4.299 , while ECPD is the least, at $2.373 .{ }^{8}$ Generally speaking, the standard errors for these estimates is a function of the amount of data. LAPP and ECPD, which have the least coverage, also have the largest standard errors. ACLED and SCAD, too, have considerably reduced coverage and contain larger standard errors.

\begin{tabular}{lrrrr}
\hline Item & $\alpha$ & $S E(\alpha)$ & $d$ & $S E(d)$ \\
\hline acled & 1.887 & 0.698 & 1.535 & 0.418 \\
banks & 2.288 & 0.26 & -1.092 & 0.117 \\
ecpd & 1.28 & 0.746 & 2.373 & 0.51 \\
idea & 1.491 & 0.432 & -0.275 & 0.104 \\
lapp & 1.234 & 1.615 & -0.076 & 1.116 \\
mec & 1.666 & 0.279 & -4.299 & 0.352 \\
regan & 1.28 & 0.259 & -1.275 & 0.132 \\
scad & 1.741 & 0.605 & 1.518 & 0.364 \\
speed & 2.11 & 0.293 & -2.404 & 0.228 \\
\hline
\end{tabular}

Table 3: All Year Binary Estimates

\section{The Graded Response Model}

The graded response model (GRM) is an extension of the 2PL to items with ordered responses, or ordinal data (Samejima 1997). The general intuition behind the GRM is to deconstruct the ordered response into a series of binary responses and estimate a $2 \mathrm{PL}$ for each. Recall in a $2 \mathrm{PL}$ model, $P\left(x_{i j}=1 \mid \theta\right)$ is estimated, where $i$ is the country-year and $j$ is the item. For the GRM, a series of 2 PL models are estimated for the $P\left(x_{i j} \geq 0 \mid \theta\right)$, the $P\left(x_{i j} \geq 1 \mid \theta\right)$, and so forth.

Let each protest measure $j$ have $C_{j}$ categories. The $d$ parameter is now $d_{1}, \ldots, d_{(C-1)}$, and represents thresholds between the ordinal responses rather than a single difficulty estimate. The discrimination parameter, $\alpha$, remains the same. The GRM is shown in Equation 2.

\footnotetext{
${ }^{8}$ Notice that the signs on the difficulty estimates are in the opposite direction, so the interpretation is reversed from what is shown in Table 3.
} 


$$
\begin{aligned}
P\left(x_{i j} \geq 0 \mid \theta_{i}\right) & =1 \\
P\left(x_{i j} \geq 1 \mid \theta_{i}\right) & =\frac{1}{1+\exp \left[-D \alpha\left(\theta_{i}-d_{1}\right)\right]} \\
P\left(x_{i j} \geq 2 \mid \theta_{i}\right) & =\frac{1}{1+\exp \left[-D \alpha\left(\theta_{i}-d_{2}\right)\right]} \\
\vdots & \\
P\left(x_{i j} \geq C_{j} \mid \theta_{i}\right) & =0
\end{aligned}
$$

In the $2 \mathrm{PL}$ model, we estimate the probability of a country-year to receive a score of ' 1 ' in the data, given its estimated quantity of protest. In the GRM, we estimate the same probability, but do so for each level of the ordinal response variable. With respect to the parameters that describe the IRFs, $\alpha_{j}$, the discrimination parameter, has a similar interpretation to that of the 2PL model. However, instead of a single difficulty parameter, a set of threshold parameters are estimated, equal to the number of categories for a given item minus one. Similar to the difficulty parameter for the 2PL model, the threshold estimates are the points at which the slope is the steepest - where there is a 0.5 probability of observing a category at or below $d_{c}$.

To estimate the probability of a country-year scoring a certain level of protest $(k)$, we subtract $k$ from $k-1$, given $\theta$. For each item, this produces a set of option response functions (ORFs), as shown in Figure 4. Ideally, the ORFs would have steep peaks across values of $\theta$, and their would not be much overlap among them. This would suggest that each protest category is meaningful and contains information that distinguishes it from other protest categories. In general, this is not observed.

The ORFs for this model demonstrate that for all indicators, the probability of observing no protest is high for significant portions of $\theta$. For MEC, the probability of observing no protest is high for nearly the entire range of $\theta$, and never reduces to zero. This is consistent with the interpretation from the binary model that MEC is a conservative protest measure. The probability of observing no protest in the ECPD data, by contrast, reduces to zero at a $\theta$ of just above zero, again consistent with the binary model that suggests ECPD is a liberal protest measure. For most items, two categories - 'no protest' and 'most protest' - are the most influential across ORFs, which is not what would be observed in the ideal case.

There are some examples of steep trace lines with little overlap in Banks, SPEED, and ACELD, but this ideal is neither the case for all levels nor for all indicators. ECPD, for example, shows that there is considerably high probability of observing categories three through seven for a large range of $\theta$. This suggests there is not much of a difference in being coded a level ' 3 ' or a level '7.' However, ECPD also shows that the middle categories are different from the 'no protest' and 'most protest' categories. Examples of less-ideal situations are Regan and SCAD. Here, not only is there considerable overlap in all categories except for the lowest and highest, but the trace lines are considerably flat and represent low densities. This suggests that not only is there little difference in the middle levels of protest, but that changes in the latent quantity of protest have very little effect on the probability of observing these levels.

The IIFs of the GRM have a similar interpretation to that of the $2 \mathrm{PL}$, but the appearance is slightly different. Due to the number of threshold estimates, each IIF can potentially contribute high levels of information across a broad range of $\theta$. An example of this is seen with ECPD, which 


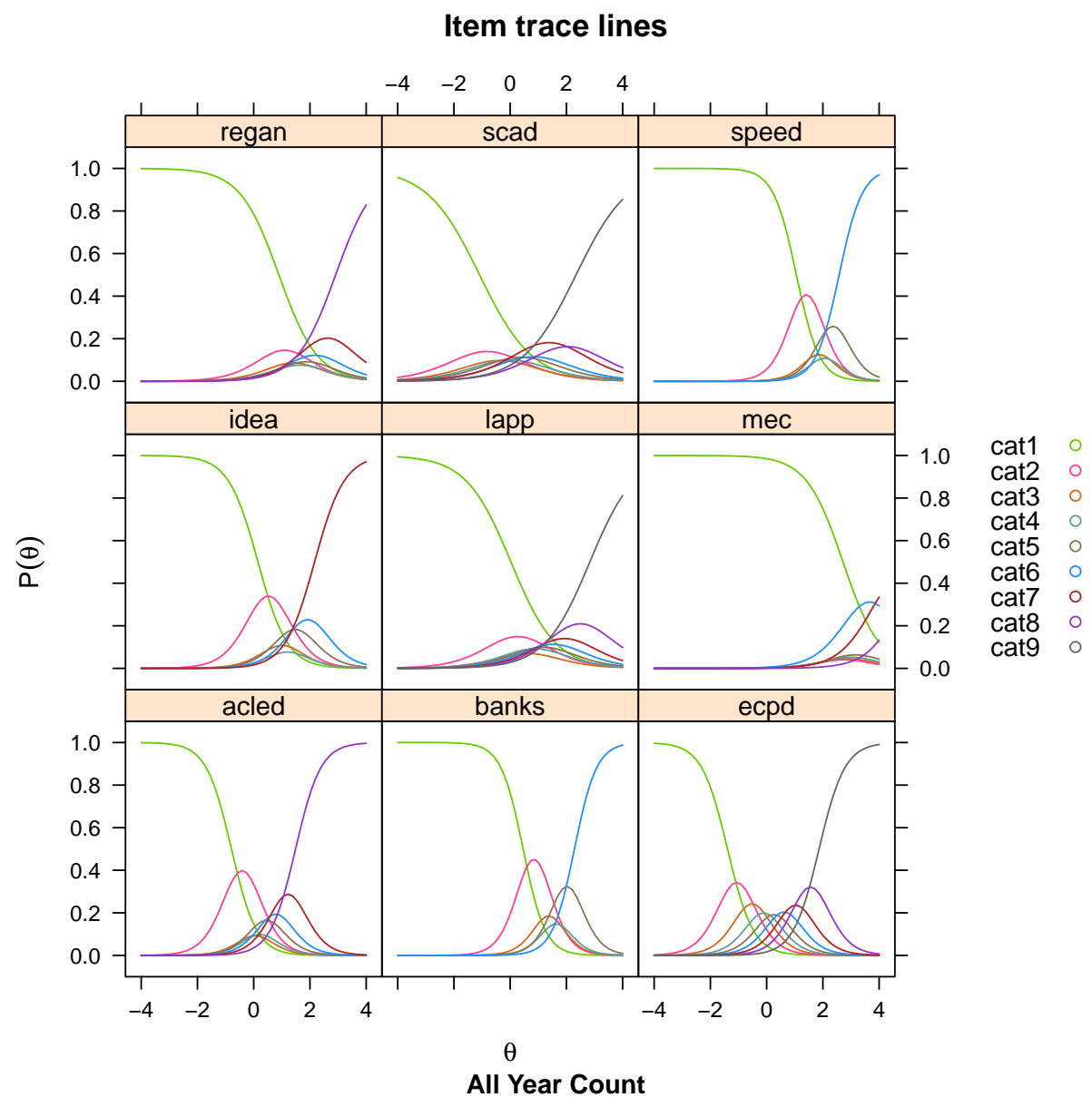

Figure 4: ORF: All Yearly Counts

contributes relatively high degrees of information over the interval of -2 to 2 . Overall, however, while sightly wider than their 2PL counterparts, the IIFs show that the information tends to concentrate in small ranges of $\theta$.

As with the 2PL, Banks is the largest contributor of information and is especially influential at the mid-range values of $\theta$. SPEED overlaps with Banks considerably, and is the second largest contributor of information. MEC continues to contribute at highest levels of $\theta$, while ACLED and ECPD are shown to be quite similar, and contain the third and fourth most information, respectively. SCAD, on the other hand, contributes almost no information in this model.

SCAD is perhaps the most detailed and well-documented of all these datasets, so its lack of information is surprising. While this is not a reflection on the validity or reliability of the SCAD data, it is a reflection on its consistency with other protest measures. For example, in both models MEC contributes at high values of $\theta$. This means that when protest is observed in the MEC data, it is likely to be observed in the other datasets as well. However, an observed protest in other datasets does not necessarily mean it will be observed in MEC. At the count level, SCAD is not consistent with other protest measures, meaning that increasing (or decreasing) levels in SCAD do not consistently correspond with increasing (or decreasing) levels in other measures. 
From the data-collection perspective, there are several possibilities as to why this low level of information is observed from SCAD in the count models. One might be that SCAD codes news reports so closely that it reflects reporting bias more precisely than other indicators. Another reason could be that SCAD's method for distinguishing its start and end dates differ from other measures. This may, for example, skew the data towards increased counts for low level protests that last for long periods of time. A third reason might be that SCAD is too liberal (in comparison to others) in its coding of individual protests. That is, where others observe a single protest, SCAD potentially observes many.

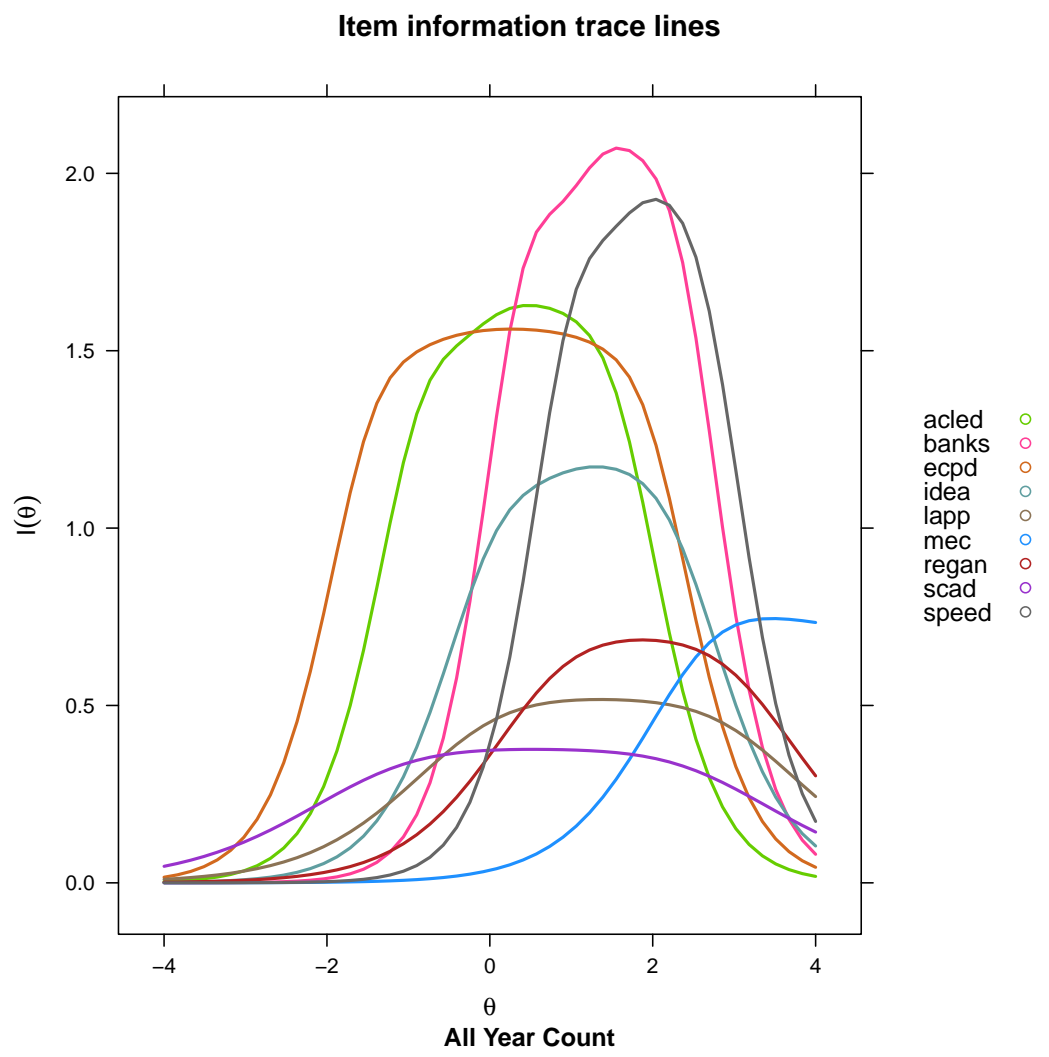

Figure 5: IIF: All Yearly Counts 


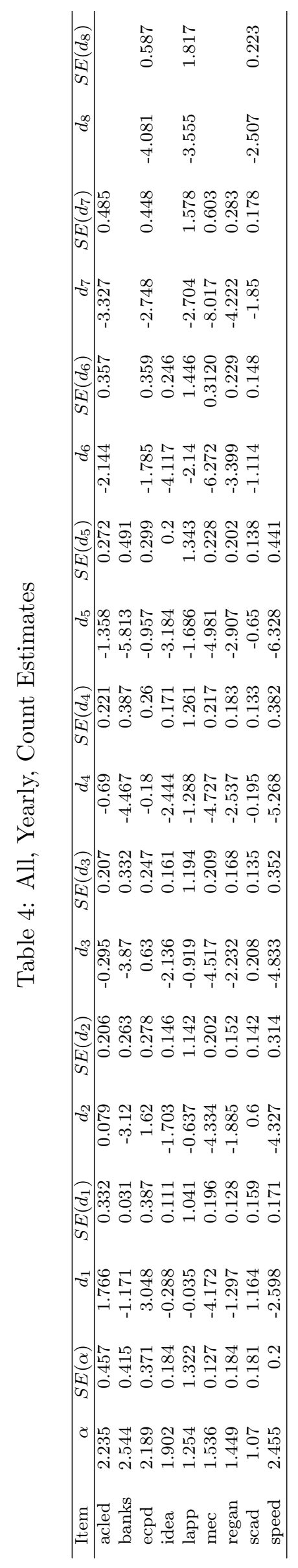


Table 4 shows the estimates for the "All Year Count" model. For each item, in addition to the discrimination parameter and its confidence interval, there is also an estimate for threshold parameters - up to eight are possible - and their associated standard errors. LAPP and SCAD have the lowest ability to discriminate at 1.254 and 1.07, respectively. However, the standard error for the LAPP estimate is relatively high (1.322), suggesting more data is necessary to accurately estimate the parameters, while SCAD's standard error is quite low (0.181). This suggests there is a high degree of confidence that SCAD is not consistent with other measures. As in the 2PL, Banks discriminates best (2.544), although its standard error is slightly larger than most others (0.415). SPEED has the next highest discrimination (2.455), and a lower standard error (0.2).

The threshold estimates take on a slightly different meaning for the GRM, because it is of interest to see if and where the categories are valuable. The initial threshold, $d_{1}$, shows that these items have a variety of levels of difficulty - a good sign that the combination of the indicators is meaningful. The largest changes in the threshold estimates occur at the first and last intervals, suggesting 'no protest' and 'most protest' are the most distinct levels. Corresponding to decreasing amounts of data, the trend for each item's standard errors is to increase as the level of protest increases. This holds for the 'most protest' level, and so the analysis of its contribution to the model should be tempered by the fact that it has the largest standard errors.

\section{Trends in Global Protest: The Value of the Latent Dimension Il- lustrated}

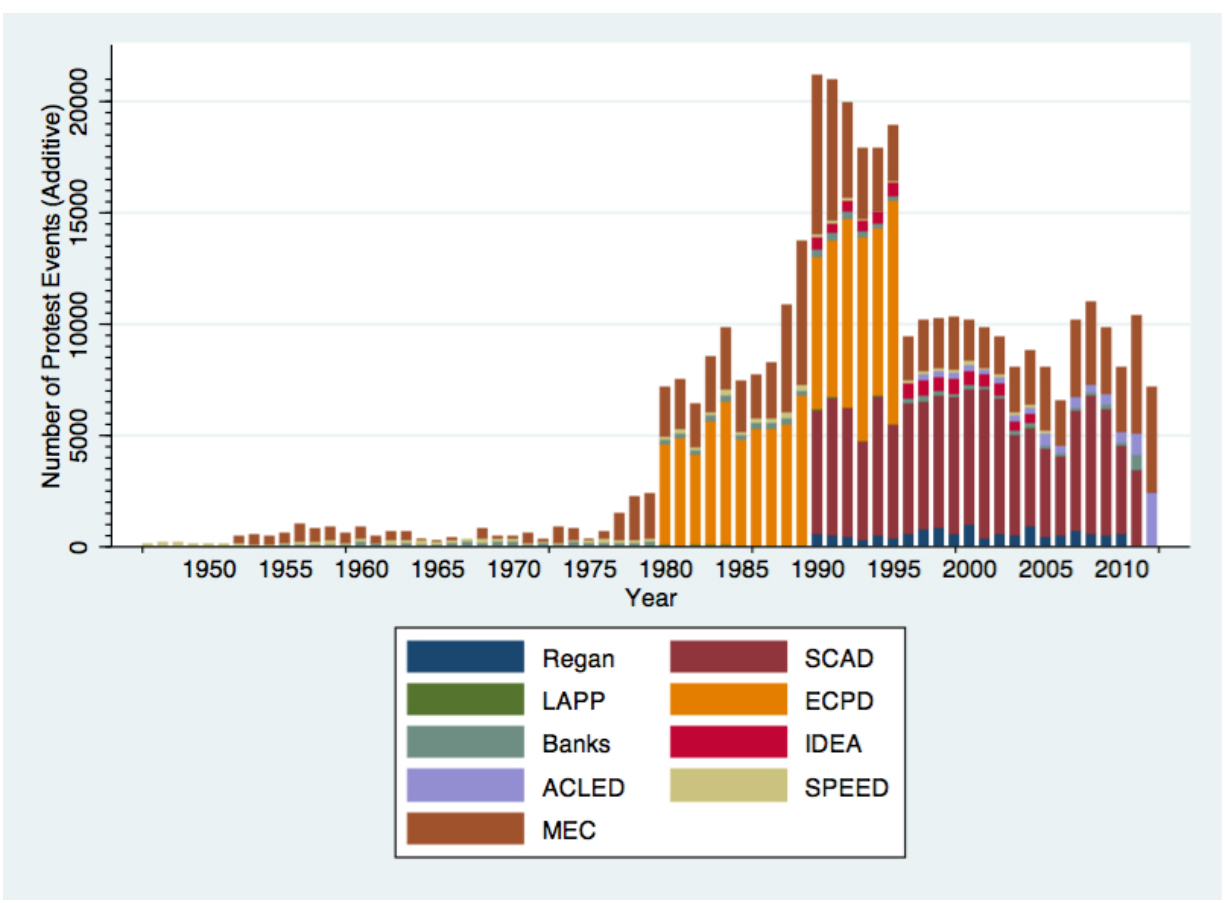

Figure 6: Global Reported Events, 1946-2012

Figure 6 illustrates the number of annual reported protests identified by each of the nine datasets. Because several datasets may report duplicate events, the y-axis should be seen as an 

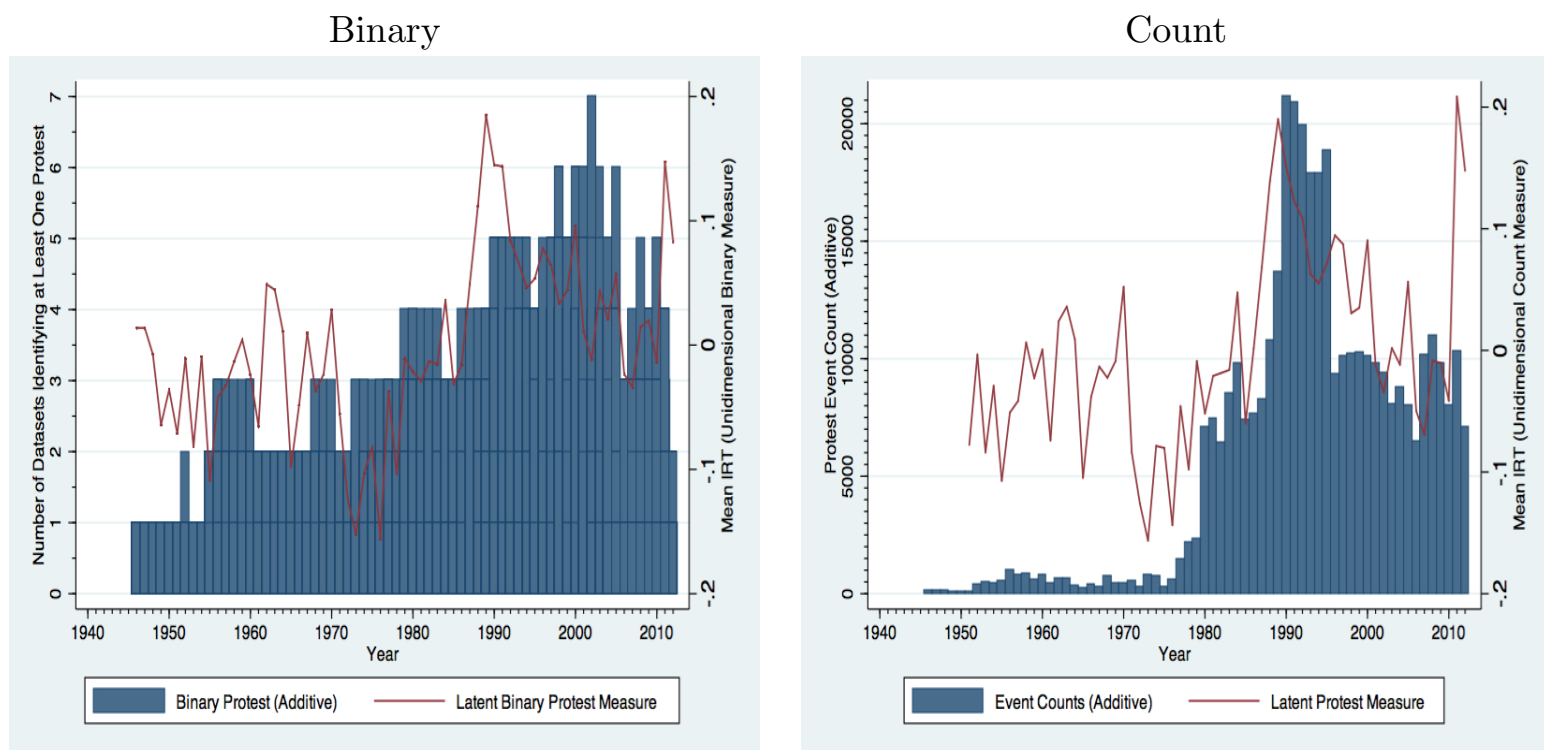

Figure 7: Global Protest with Mean IRT, 1946-2012

additive set of protest event counts rather than a raw count of protest events. Nonetheless, as is clear from this figure, with the exception of Banks, MEC, and SPEED most protest datasets do not begin coverage until 1980. Moreover, some of the datasets with the highest quantity of reported events after 1980 have limited geographical and temporal coverage. ECPD, which focuses exclusively on Europe, ends coverage in 1995, yielding a sharp decline in the number of reported events. SCADs coverage appears in 1990, yielding a high number of reported events but for Africa only. The difference in spatial and temporal coverage over time illustrates one key challenge in estimating the quantity of protest. Another key challenge is the fact that two of the protest databases with the greatest scope of geographical and temporal coverage (e.g. Banks and SPEED) contribute a very small proportion of the total reported events, suggesting that they significantly underreport events that other datasets capture.

To illustrate the added value of the IRT exercise, we identify how the binary and count latent measures might add information to observed global trends. The left panel of Figure 7 identifies the number of datasets with at least one observed protest in a given year - an additive count of binary measures of protest. 2005 was the year with the best coverage, since 7 of the 9 datasets identified at least one protest in that year. We can see, however, that the mean of our latent binary protest variable indicates higher levels of potential protest in 1946-1954 than was observed. This is also true in the early-mid 1960s, as well as in 1988-1990 (during the height of the Eastern European revolutions) and 2011 (the height of the Arab Spring).

These patterns are equally striking when we observe the global reported event counts compared with the mean of our latent count variable (right panel of Figure 7 ). Here again, we see a much higher latent potential for protest than was observed during the entire 1950-1989 period, and a much higher latent potential for protest than was reported in 2011.

Returning to the Tunisia case, Figure 8 illustrates the additive event counts from the different available data sets from 1946-2012 (excluding LAPP). Once again, we can see from the left panel Figure 9 that in general, the latent variable for binary protest provides more information than the observed measures in the 1946-1989 period. Interestingly, the latent measure is quite consistent with 


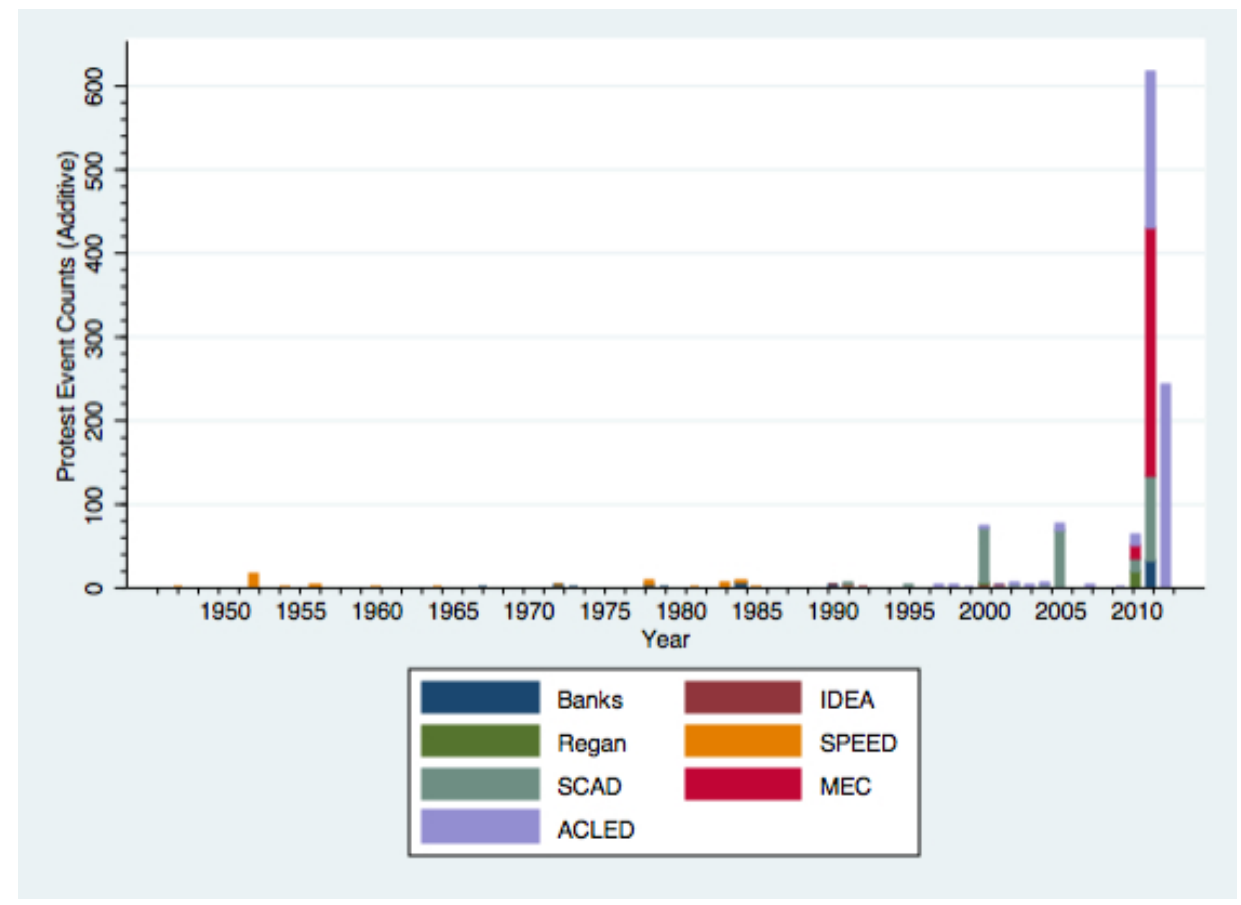

Figure 8: Tunisia Reported Events, 1946-2012

the observed binary indicators after 1989. The count variables yield more information, however. From the right panel of Figure 9, we can see that the latent potential for protest is higher than observed protest from 1950-2005. Moreover, the latest measure properly categorizes 2010-2012 as highly contentious years in Tunisian politics.

In summary, both models appear to estimate the latent quantity of protest well, with the caveat that the GRM is not much more informative than the $2 \mathrm{PL}$, if it is more informative at all. Rather than more accurately reflect a quantity of protest, counts of protest may obscure that latent quantity, as is seen in the SCAD example. The test put forth in the following section is more revealing than a descriptive comparison of the estimates. In the next section, the ability of the newly estimated quantity of protest is tested against a well-known forecasting model that has been originally estimated using just the Banks measure.

\section{Authoritarian breakdown}

To illustrate one use of the estimated protest data, in this section we present results from authoritarian breakdown models that include this variable as a predictor. The authoritarian breakdown data are from an updated version of Geddes, Wright and Frantz (2014). The updates include data from 1946 to 2012 on 286 autocratic regimes in 119 countries. We examine the post-Cold War period, from 1991-2012, during which 73 autocratic breakdown events occur: 50 democratic transitions and 23 regime breakdown events where the subsequent regime is not a democracy (what we call autocratic transition). The latter transitions entail one autocratic regime replacing another, 
Binary

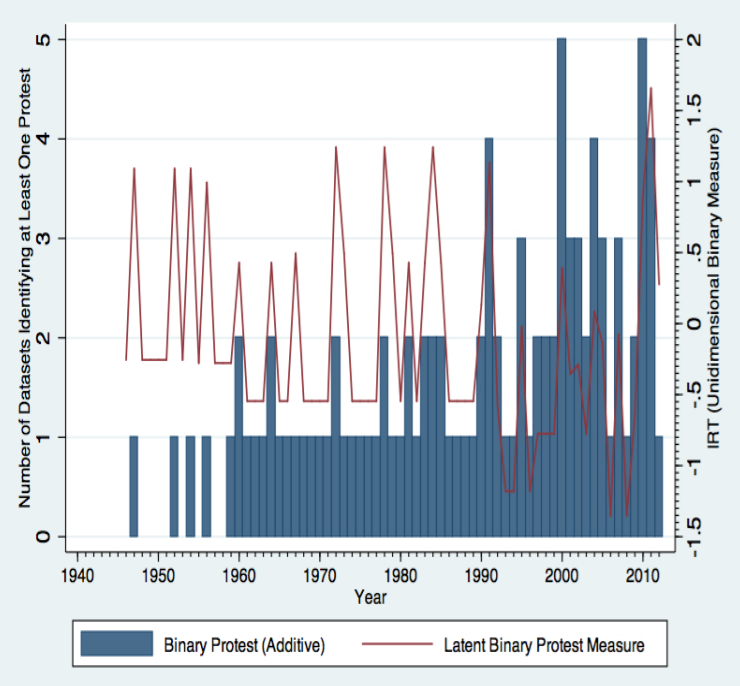

Count

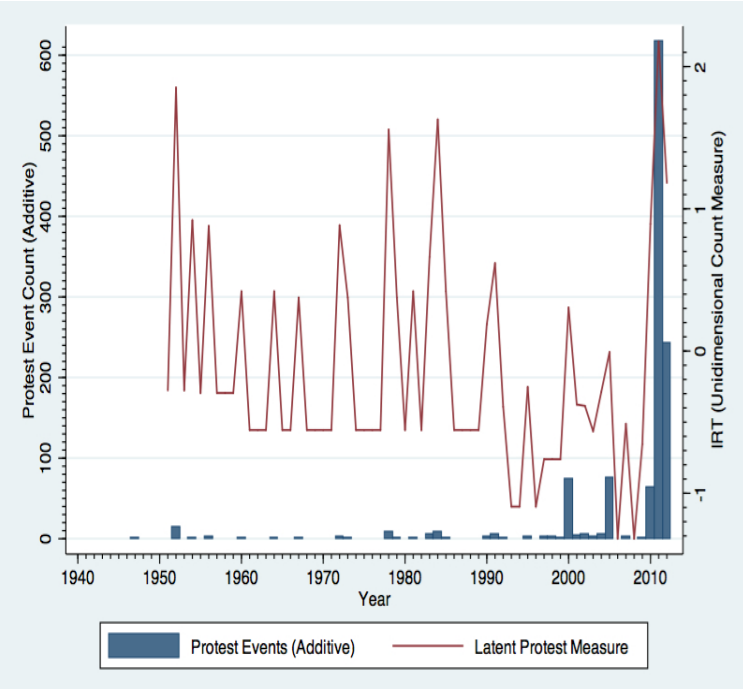

Figure 9: Tunisia Protest with Binary IRT Measure, 1946-2012

for example Laurent Kabila's rebel army ousting the Mobutu regime from Kinshasa in 1997. ${ }^{9}$

We begin with yearly protest data so that we can examine how well the latent measure performs relative to the most widely used extant measure from the Banks data set. First, we test and validate a model using data from 1991-2012 for three dependent variables: all breakdowns, democratic transitions, and autocratic transitions. Below we describe the model specification for each dependent variable. We estimate a Weibull survival model. ${ }^{10}$

To validate the model, we conduct out-of-sample tests, following Ward, Greenhill and Bakke (2010). We randomly divide the sample into 10 bins and run the model with data from 9 of the 10 bins, using data from the tenth for out-of-sample prediction. We do this ten times, each time saving data from a different bin to conduct out-of-sample predictions. We obtain predictions from the survivor function by substracting the bounded predicted survival rate from $1: 1-\hat{S}$. This provides us with one out-of-sample prediction for all data in the sample. We conduct this procedure ten times (randomizing differently each time) and average the ten out-of-sample predictions. We then calculate the AUC for the averaged out-of-sample predictions, both in a model with the protest information and one without them. For each dependent variable, we choose a model specification with the highest AUC for these averaged out-of-sample predictions.

Table 5 reports the out-of-sample AUCs for each model (we discuss covariate data and report the estimates for covariates in the Appendix). In each case, we choose a different model specification, including a different protest variable. For example, the All breakdowns model uses a one-year lag

${ }^{9}$ This latter category also includes regime failures where the subsequent regime is either an occupying foreign power (e.g. Iraq 2003) or a power vacuum exists such that no internationally recognized government controls the majority of territory (e.g. Somalia after the fall of Siad Barre in 1991).

${ }^{10}$ The hazard rate at time $t$ in this model is $h(t, X)=\lambda p(\lambda t)^{p-1}$, where $\lambda_{i}=e^{X_{i} \beta}$. X is the vector of explanatory variables and $p$ is a shape parameter that estimates how the hazard changes over time. 
on the protest measure derived from binary protest data (any vs. none), whereas the Democratic transition model employs the 2-year lagged moving average of the latent protest measure derived from the binary protest data. The set of variables from which we choose are theoretically informed, but the variables specificied in each model, including the protest variable(s), are based on the specification that yields the highest average AUC score from the out-of-sample validation. For All breakdowns and Democratic transitions, adding the protest information from the latent measure increases the validation AUCs by over 0.02. This means that protest information increases the predictive accuracy of these models. For the Autocratic transition model, however, adding protest information does not change the predictive accuracy of the model.

Table 5: Out-of-sample tests, yearly data

\begin{tabular}{lcccc}
\hline \hline & & & & \\
Model & $\begin{array}{c}\text { Number } \\
\text { of Events }\end{array}$ & $\begin{array}{c}\text { AUC w/out } \\
\text { Protest }\end{array}$ & $\begin{array}{c}\text { AUC with } \\
\text { Protest }\end{array}$ & Difference \\
\hline $\begin{array}{l}\text { All breakdowns } \\
\text { Latent protest (binary, one-year lag) }\end{array}$ & 73 & 0.728 & 0.765 & 0.037 \\
\hline $\begin{array}{l}\text { Democratic transition } \\
\text { Latent protest (binary, lagged 2-year MA) }\end{array}$ & 50 & 0.802 & 0.827 & 0.025 \\
\hline $\begin{array}{l}\text { Autocratic transition } \\
\text { Latent protest (binary, one-year lag) }\end{array}$ & 23 & & & \\
\hline \hline
\end{tabular}

1991-2012; 129 regimes in 92 countries.

Figure 10 shows the separation plots for the out-of-sample predictions for the three models in Table 5. While there are some clear misses in the democratic transition separation plot (Azerbaijan 1992, Liberia 2003), it performs betterthan the autocratic transitions model, particularly at the high end of the instability rank axis.

Table 6: Comparing the Latent and Banks measures, yearly data

\begin{tabular}{lcccc}
\hline \hline Model & $\begin{array}{c}\text { Number } \\
\text { of Events }\end{array}$ & $\begin{array}{c}\text { Banks } \\
\text { Protest }\end{array}$ & $\begin{array}{c}\text { Latent } \\
\text { Protest }\end{array}$ & Difference \\
\hline & & \multicolumn{2}{c}{ In-sample AUCs } & \\
All breakdowns & 73 & 0.789 & 0.797 & 0.008 \\
Democratic transition & 50 & 0.849 & 0.862 & 0.013 \\
Autocratic transition & 23 & 0.788 & 0.791 & 0.003 \\
& & & & \\
& & & & \\
All breakdowns & 7 & 0.597 & 0.605 & 0.008 \\
Democratic transition & 6 & 0.567 & 0.652 & 0.085 \\
Autocratic transition & 1 & & & \\
\hline \hline
\end{tabular}

1991-2012; 129 regimes in 92 countries.

Next we compare each model that uses the latent measure with a similar model that employs the Banks data. Again, we used out-of-sample validation to choose the Banks protest variable 


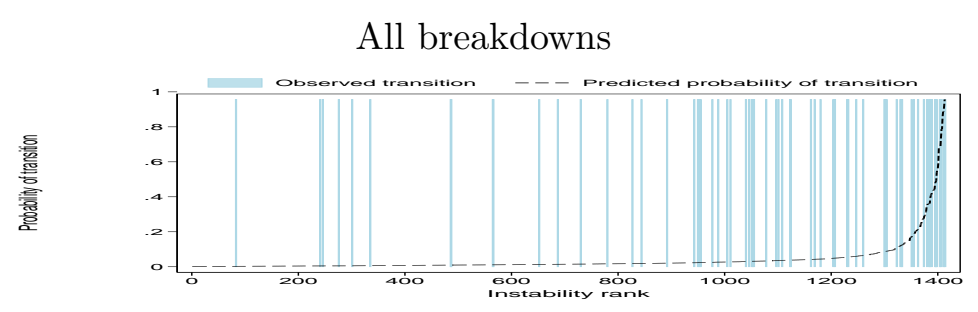

Democratic transitions

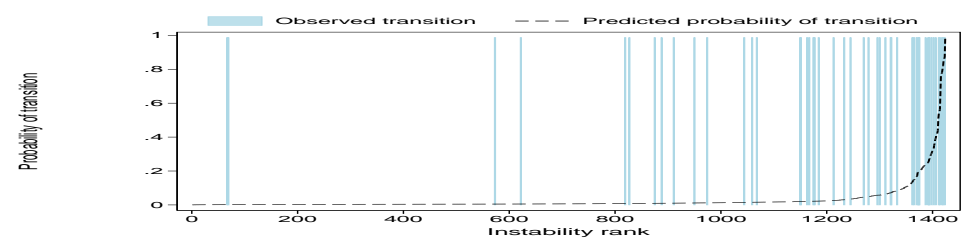

Autocratic transitions

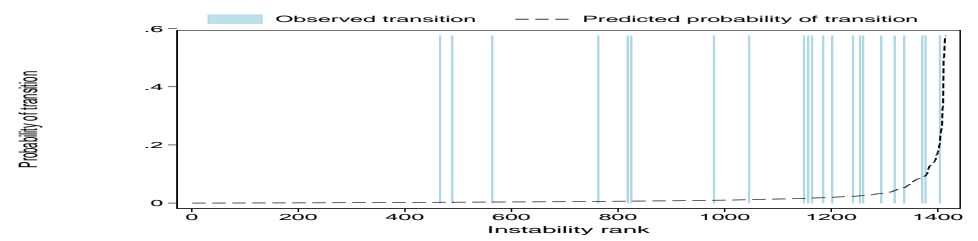

Figure 10: Separation plots for out-of-sample validation (yearly). Years: 1991-2012. See Table 5 details on protest variables.

wth the highest average out-of-sample AUC scores. We also restrict the sample to all observations with non-missing data on both measures of protest. First, we compare the in-sample AUC scores from models with each of the protest measures. The top panel of Table 6 shows that for each dependent variable, the latent measure increases the predictive accuracy of the model in-sample, relative to the Banks measure. The relative increase in predictive accuracy, however, is largest for the Democratic transition model and neglible for the Autocratic transition model. Second, we estimate the model with data from 1991-2010 and gauge the predictive accuracy of the model against observed autocratic breakdowns in the years 2011-12. We only do this for All breakdowns and for Democratic transition because there is only one observation of Autocratic breakdown in those two years. Recall that an AUC score of 0.50 is no better than chance, so overall these forecasts perform poorly relative to the validation and in-sample AUC tests. That said, the forecast using the latent measure does appreciably better than the forecast using the Banks measure, suggesting some value-added to using the latent measure of protest in a predictive model of democratic transition.

\section{Monthly data}

A second potential advantage of the latent measure is to utilize the monthly data. Again we examine a post-Cold War sample (1990-20102) and three dependent variables: All breakdowns, Democratic transitions, and Autocratic transitions. ${ }^{11}$ During these 23 years here are 80 breakdown events (56 democratic transitions and 24 autocratic transtions) and over 17,000 non-events months.

\footnotetext{
${ }^{11}$ With the yearly data and the one-year lag on the protest variables, the year 1990 was dropped from the analysis.
} 
We test and validate a model for each dependent variable to maximize the out-of-sample AUC scores, as described above. In addition to the monthly data on protest and regime breakdwon, we utlize monthly data on coups, civil and international conflict, (and elections... not yet!). Data for autocratic regime type and prior democracy vary by regime; and data on infant mortality rates vary by year. The covariates are: successsful Coup in the past 24 months; Prior democracy; IMR relative to the world mean; Military regime; Monarchy; Protest; Military $\times$ Protest.

For each model (dependent variable, sample) we conducted out-of-sample tests, similar to those conducted using the yearly data. The first column of Table 7 reports the average AUC from these tests without the protest variables, for each of the models (dependent variables). The second column reports the average out-of-sample AUCs from validation tests with the protest variables, while the third column shows the difference between the average AUCs in the first and second columns. While the democratic transition models has the best predictive accuracy, including protest information increases the out-of-sample AUCs for all the models.

Next we examine the forecast for the months during the calendar years 2011-12. To do this, we test the model for each dependent variable with data from 1990-2010 and then calculate predictions for the months in years 2011-12. We then assess these forecasts with observed monthly data on regime breakdown in 2011-12. The AUCs from these tests are reported in the last column of Table 7. Again the forecast for democratic transition has a higher AUC than the forecast for all breakdowns, but overall the 2011-12 forecasts are substantially better with the monthly data than with the yearly data. For example the democratic transition forecast AUC is 0.742 with the monthly data but only 0.652 with the yearly data. Further, the monthly model does not yet have the monthly election data).

Table 7: Out-of-sample tests, monthly data

\begin{tabular}{lccccc}
\hline \hline & $\begin{array}{c}\text { Number } \\
\text { of Events }\end{array}$ & $\begin{array}{c}\text { AUC w/out } \\
\text { Protest }\end{array}$ & $\begin{array}{c}\text { AUC with } \\
\text { Protest }\end{array}$ & Difference & $\begin{array}{c}\text { Forecast } \\
\text { AUC }\end{array}$ \\
\hline $\begin{array}{l}\text { All breakdowns } \\
\text { Count protest (lagged 4-month MA) }\end{array}$ & 80 & 0.676 & 0.744 & 0.068 & 0.692 \\
\hline $\begin{array}{l}\text { Democratic transition } \\
\text { Count protest (lagged 6-month MA) }\end{array}$ & 56 & 0.712 & 0.788 & 0.076 & 0.742 \\
\hline $\begin{array}{l}\text { Autocratic transition } \\
\text { Count protest (lagged 2-month MA) }\end{array}$ & 24 & 0.542 & 0.617 & & \\
\hline \hline
\end{tabular}

137 regimes in 96 countries from 1990-2012.

It is important to note that the lagged moving averages for protest that yield the best predictions are 2-, 4-, and 6-month MAs. This means that moving to monthly data on protests yields better model predictions than simply using lagged yearly averages for protest, even with data that models these monthly outcome variables. Further, when we test the forecast model for democratic transitions and use the 2-month lagged MA instead of the 6-month lagged MA, the AUC for the 2011-12 forecast period is 0.804, which suggests that an even smaller time frame for protest measures may have helped predict autocratic regime breakdown at the monthly level during the 2011-12 
period. $^{12}$

Finally, the next iteration of the monthly models will include monthly election data as well as test split population survival models to examine whether structural features (e.g. slowly moving infant mortality rates) are best modeled that way.

\section{Conclusion}

The latent protest variable produced in this analysis performs better in-sample and with models of democratic transition. At this stage, therefore, the added value of this variable is certainly present, although other datasets also perform fairly well overall.

This exercise tells us a few things about current protest databases. First, including protest variables in models of authoritarian breakdown improves both in-sample and out-of-sample forecasts of this phenomenon - both in terms of all autocratic breakdown events grouped together and democratic transitions. This finding highlights the importance of protest as a covariate and further underscores the necessity of reliable measurement of this indicator. Second, measurement differences across datasets - as modeled by our latent protest indicator - produce nontrivial differences in the forecasts of different transition types, both in- and out-of-sample. Although the results were somewhat mixed, where our latent variable performed the best (in terms of democratic transitions), its impact was substantially different from that of different protest databases. As a consequence, our study illustrates the potential value of modeling the latent dimensions of protest rather than solely relying on observed indicators.

\footnotetext{
${ }^{12}$ For all breakdowns, the forecast AUC with a 2-month lag MA is 0.719.
} 


\section{References}

Baker, Frank B. 2001. The Basics of Item Response Theory. Second ed. New York: ERIC Clearinghouse.

Baker, Frank B. and Seoch-Ho Kim. 2004. Item Response Theory: Parameter Estimation Techniques. Second ed. New York: Marcel Dekker.

Benson, Brett V. and Joshua D. Clinton. 2012. "Measured Strength: Estimating the Strength of Alliances in the International System, 1881-2000." Unpublished manuscript.

Chalmers, R. Philip. 2012. "mirt: A Multidimensional Item Response Theory Package for the R Environment." Journal of Statistical Software 48(6).

Clinton, Joshua D. and Simon Jackman. 2009. "To Simulate or NOMINATE?" Legislative Studies Quarterly 24(4):593-621.

D'Orazio, Vito. 2013. International Military Cooperation: From Concepts to Constructs PhD thesis Pennsylvania State University.

Geddes, Barbara, Joseph Wright and Erica Frantz. 2014. "Autocratic Regimes: A New Data Set." Perspectives on Politics 17(1):forthcoming.

Herkenrath, Mark and Alex Knoll. 2011. "Protest events in international press coverage: An empirical critique of cross-national conflict databases." International Journal of Comparative Sociology 52(3):163-180.

Hyde, Susan and Nikolay Marinov. 2012. "Which Elections Can Be Lost?" Political Analysis 20(2):191-210.

Linden, Wim J. van der and Ronald K. Hambleton. 1997. Item Response Theory: Brief History, Common Models, and Extensions. In Handbook of Modern Item Response Theory, ed. Wim J. van der Linden and Ronald K. Hambleton. Springer New York pp. 1-28.

Marshall, Monty G. and Keith Jaggers. 2007. "Polity IV Project: Political Regime Characteristics and Transitions 1800-2007.”. Center for Systemic Peace, George Mason University.

McAdam, Doug. 1999. Political process and the development of black insurgency, 1930-1970. University of Chicago Press.

Pemstein, Daniel, Stephen A Meserve and James Melton. 2010. "Democratic compromise: A latent variable analysis of ten measures of regime type." Political Analysis 18(4):426-449.

Pieroni, Luca, Giorgio d'Agostino and Francesco Bartolucci. 2013. "Identifying corruption through latent class models: evidence from transition economies.".

Powell, Jonathan M and Clayton L Thyne. 2011. "Global instances of coups from 1950 to 2010 A new dataset." Journal of Peace Research 48(2):249-259.

Reise, Steven P. and Niels G. Waller. 2009. "Item Response Theory and Clinical Measurement." Annual Review of Clinical Psychology 5:27-48. 
Samejima, Fumiko. 1997. Graded Response Model. In Handbook of Modern Item Resonse Theory, ed. Wim J. van der Linden and Ronald K. Lambleton. Springer.

Scott, James C. 1985. Weapons of the weak: Everyday forms of peasant resistance. Yale University Press.

Smith, Jackie, John D McCarthy, Clark McPhail and Boguslaw Augustyn. 2001. "From protest to agenda building: Description bias in media coverage of protest events in Washington, DC." Social Forces 79(4):1397-1423.

Toland, Michael D. 2013. "Practical Guide to Conducting an Item Response Theory Analysis." The Journal of Early Adolescence pp. 1-32.

Treier, Shawn and Simon Jackman. 2008. "Democracy as a latent variable." American Journal of Political Science 52(1):201-217.

Ward, Michael D, Brian D Greenhill and Kristin M Bakke. 2010. "The perils of policy by p-value: Predicting civil conflicts." Journal of Peace Research 47(4):363-375. 


\section{Variable sources}

Armed Conflict Location and Event Dataset (acled)

Downloaded from: https://www.strausscenter.org/acled.html on 9.12.13.

Version: ACLED All Africa 1997-November 2013.

Data structure: daily event; each row records event that occurs for no longer than 1 day

Cross-National Time-Series Data Archive (banks)

Downloaded from: www.databanksinternational.com on 9.1.13.

Version: data available on retrieval date

Data structure: country-year

European Protest and Coercion Data from Ronald Francisco (epcd)

Downloaded from: http://web.ku.edu/ ronfrand/data/index.html on 9.1.14.

Version: data available on download date.

Data structure: daily event; each row records event that occurs for no longer than 1 day

Integrated Data for Event Analysis (idea)

Downloaded from: http://thedata.harvard.edu/dvn/ on 2.12.13.

Version: http://hdl.handle.net/1902.1/FYXLAWZRIA UNF : 3:dSE0bsQK206xXlxeaDEhcg==

IQSS Dataverse Network [Distributor] V3 [Version]

Data structure: daily event; each row records event that occurs for no longer than 1 day

Latin American Political Protest Project (lapp)

Downloaded from: http://faculty.mwsu.edu/politicalscience/steve.garrison/LAPPdata/ on 9.12.13.

Version: data available on download date.

Data structure: daily event; each row records event that occurs for no longer than 1 day

Major Episodes of Contention Data Project (mec)

Obtained from Erica Chenoweth on 4.1.14.

Version: MEC Cat4 1950-2013.

Data structure: event; each row records event that occurs for multiple days to years

Protest and State Response Data (regan)

Obtained from PITF on 1.9.13.

Version: June 2012.

Data structure: event; each row records event that occurs for possibly multiple days

Social Conflict in Africa Database (scad)

Downloaded from: https://www.strausscenter.org/scad.html on 9.12.13.

Version: SCAD 3.0 1990-2011.

Data structure: event; each row records event that occurs for possibly multiple days

Social, Political and Economic Event Database Project (speed)

Downloaded from: http://www.clinecenter.illinois.edu/research/speed-data.html

on 2.12.13.

Version: data available on retrieval date

Data structure: daily event; each row records event that occurs for no longer than 1 day 


\section{Variable definitions}

- acled_protest_any (binary)

Events include: "Riots/Protests" OR ["Non-violent activity by a conflict actor" AND [ "Civilians" OR "Protestes" OR "Rioters" as actors]]

Variable values:

0. no protest

1. protest

- acled_protest_count (count)

Events include: "Riots/Protests" OR ["Non-violent activity by a conflict actor" AND [ "Civilians" OR "Protestes" OR "Rioters" as actors]]

Variable values:

- number of protests, including zero

- banks_protest_any (binary)

Events include: "Riots" OR "Strikes" OR "Anti-government demonstrations" OR "Nonviolent action"

Variable values:

0 . no protest

1. protest

- banks_protest_count (count)

Events include: "Riots" OR "Strikes" OR "Anti-government demonstrations" OR "Nonviolent action"

Variable values:

- number of protests, including zero

- ecpd_protest_any (binary)

Events include: "accede" OR "adaptation" OR "agreement" OR "ambush" OR "appeal" OR "backlash" OR "battle" OR "blockade" OR "boycott" OR "breakout" OR "break-in" OR "cancel" OR "' OR "censor" OR "ceremony" OR "civil disobedience" OR "closure" OR "commemorate" OR "commitment" OR "compromise" OR "confiscate" OR "confront" OR "conscription" OR "convict" OR "convoy" OR "counter-demonstration/rally" OR "curfew" OR "declare" vOR "defection" OR "defuse" OR "delay" OR "demolish" OR "demonstrate" OR "deploy" OR "deport" OR "destroy" OR "disband" OR "dismiss" OR "disrupt" OR "disolve" OR "disturb" OR "elect" OR "escape" OR "exclude" OR "exit" OR "expel" OR "expulsion" OR "fine" OR "fire" OR "force" OR "funeral" OR "general strike" OR "harass" OR "hide" OR "hunger strike" OR "immolation" OR "impeach" OR "intervention" 
OR "lockout" OR "mobilize" OR "motorcade" OR "negotiation" OR "obstruct" OR "offer" OR "opposition" OR "oust" OR "petition" OR "preclude" OR "pre-empt" OR "propoganda" OR "proscribe" OR "protest" OR "raid" OR "rally" OR "refuse" OR "reject" OR "release" OR "religion mass" OR "removal" OR "repression" OR "resign" OR "riot" OR "rob" OR "sabotage" OR "satire" OR "search" OR "seize" OR "self-mutilation" OR "shut down" OR "silent protest" OR "sit-in" OR "slowdown" OR "strike" OR "suicide" OR "support" OR "surrender" OR "suspend" OR "symbolic" OR "transfer" OR "trespass" OR "trial" OR "ultimatim" OR "vigil" OR "vote" OR "walkout" OR "warn" OR "withdraw" OR "with hold" OR "work stoppage"

Variable values:

0. no protest

1. protest

- ecpd_protest_count (count)

Events include: "accede" OR "adaptation" OR "agreement" OR "ambush" OR "appeal" OR "backlash" OR "battle" OR "blockade" OR "boycott" OR "breakout" OR "break-in" OR "cancel" OR "' OR "censor" OR "ceremony" OR "civil disobedience" OR "closure" OR "commemorate" OR "commitment" OR "compromise" OR "confiscate" OR "confront" OR "conscription" OR "convict" OR "convoy" OR "counter-demonstration/rally" OR "curfew" OR "declare" vOR "defection" OR "defuse" OR "delay" OR "demolish" OR "demonstrate" OR "deploy" OR "deport" OR "destroy" OR "disband" OR "dismiss" OR "disrupt" OR "disolve" OR "disturb" OR "elect" OR "escape" OR "exclude" OR "exit" OR "expel" OR "expulsion" OR "fine" OR "fire" OR "force" OR "funeral" OR "general strike" OR "harass" OR "hide" OR "hunger strike" OR "immolation" OR "impeach" OR "intervention" OR "lockout" OR "mobilize" OR "motorcade" OR "negotiation" OR "obstruct" OR "offer" OR "opposition" OR "oust" OR "petition" OR "preclude" OR "pre-empt" OR "propoganda" OR "proscribe" OR "protest" OR "raid" OR "rally" OR "refuse" OR "reject" OR "release" OR "religion mass" OR "removal" OR "repression" OR "resign" OR "riot" OR "rob" OR "sabotage" OR "satire" OR "search" OR "seize" OR "self-mutilation" OR "shut down" OR "silent protest" OR "sit-in" OR "slowdown" OR "strike" OR "suicide" OR "support" OR "surrender" OR "suspend" OR "symbolic" OR "transfer" OR "trespass" OR "trial" OR "ultimatim" OR "vigil" OR "vote" OR "walkout" OR "warn" OR "withdraw" OR "with hold" OR "work stoppage"

Variable values:

- number of protests, including zero

- idea_protest_any (binary)

Events include: "Non-military demonstrate" (WEISS code 181, civil actor)

Variable values:

0. no protest 
1. protest

- idea_protest_count (count)

Events include: "Non-military demonstrate" (WEISS code 181, civil actor)

Variable values:

- number of protests, including zero

- lapp_protest_any (binary)

Events include:

- Bolivia: "Demonstrations" OR "Protests" OR "Riots" OR "Roadblocks" OR "Sit-ins" OR "Strikes"

- Colombia: "Demonstrations" OR "Protests" OR "Riots" OR "Roadblocks" OR "Sitins" OR "Strikes"

- El Salvador: "Demonstrations" OR "March" OR "Occupation" OR "Protests" OR "Riots" OR "Roadblocks" OR "Sit-ins" OR "Strikes"

- Peru: "Demonstrations"

Variable values:

0. no protest

1. protest

- lapp_protest_count (count)

Events include:

- Bolivia: "Demonstrations" OR "Protests" OR "Riots" OR "Roadblocks" OR "Sit-ins" OR "Strikes"

- Colombia: "Demonstrations" OR "Protests" OR "Riots" OR "Roadblocks" OR "Sitins" OR "Strikes"

- El Salvador: "Demonstrations" OR "March" OR "Occupation" OR "Protests" OR "Riots" OR "Roadblocks" OR "Sit-ins" OR "Strikes"

- Peru: "Demonstrations"

Variable values:

- number of protests, including zero

- mec_protest_any (binary)

Events include: each calendar day/month/year during which a non-violent protest campaign existed

Variable values:

0. no protest

1. protest 
- mec_protest_count (count)

Events include: each calendar day/month/year during which a non-violent protest campaign existed

Variable values:

- number of protests, including zero

- regan_protest_any (binary)

Events include: each calendar day of protest event. "Protest is a gathering of 50 or more people to express a demand for social or political access, tolerance, or resources. A protest action must be targeted at the state or state policy." Coded from search terms for "protests", "riots," and "demonstrations"

\section{Variable values:}

0. no protest

1. protest

- regan_protest_count (count)

Events include: each calendar day of protest event. "Protest is a gathering of 50 or more people to express a demand for social or political access, tolerance, or resources. A protest action must be targeted at the state or state policy." Coded from search terms for "protests" OR "riots" OR "demonstrations'

Variable values:

- number of protests, including zero

- scad_protest_any (binary)

Events include: "Organized Demonstration" OR "Spontaneous Demonstration" OR "Organized Violent Riot" OR "Spontaneous Violent Riot" OR "General Strike" OR "Limited Strike". Coded from search terms for "protest" OR "strike" OR "riot" OR "violence" OR "attack"

\section{Variable values:}

0 . no protest

1. protest

- scad_protest_count (count)

Events include: "Organized Demonstration" OR "Spontaneous Demonstration" OR "Organized Violent Riot" OR "Spontaneous Violent Riot" OR "General Strike" OR "Limited Strike". Coded from search terms for "protest" OR "strike" OR "riot" OR "violence" OR "attack"

Variable values: 
- number of protests, including zero

- speed_protest_any (binary)

Events include: "Mass demonstrations or strikes" OR "riots or brawls"

Variable values:

0. no protest

1. protest

- speed_protest_count (count)

Events include: "Mass demonstrations or strikes" OR "riots or brawls"

Variable values:

- number of protests, including zero 


\section{Yearly data}

In the Democratic transition model, we employ information from the following variables: a binary variable indicating Prior democracy; a binary indicator of a successful Coup in the past four years from Powell and Thyne (2011); a binary indicator of Military regime from Geddes, Wright and Frantz (2014); a binary indicator of an Incumbent standing in an election either the observation year or the prior year and an indicator of an international election Monitor present during the election from Hyde and Marinov (2012); and a lagged moving average of the latent Protest constructed from count information. The model specification with the highest out-of-sample AUCs from the validation also include interaction terms: Mililtary $\times$ Protest, Mililtary $\times$ Coup, Mililtary $\times$ Incumbent.

In the Autocratic transition model, we employ information from the following variables: a binary indicator of an attempted Coup in the past year from Powell and Thyne (2011); a binary indicator of an Irregular election in either the observation year or the prior year from Hyde and Marinov (2012); a continous measure of the infant mortality rate relative to the word mean $(I M R)$; and the 1-year lag of the latent Protest variable constructed from binary information. The model specification with the highest out-of-sample AUCs from the validation test also includes an interaction term: Irregular $\times \log ($ duration $)$, where the latter term is the natural log of regime duration in years.

In the All breakdowns model, we employ information from the following variables: a binary indicator of a successful Coup in the past four years; the 1-year lag of the latent Protest variable

constructed from binary information; Irregular; IMR; Prior; Military; Military $\times$ Incumbent; and Military $\times$ Coup. 
Table A-1: Authoritarian breakdown, yearly

\begin{tabular}{|c|c|c|c|}
\hline & All & Dem & Dict \\
\hline Protest & $\begin{array}{c}1.267^{* *} \\
(0.46)\end{array}$ & $\begin{array}{l}1.182 \\
(0.62)\end{array}$ & $\begin{array}{l}0.644 \\
(0.67)\end{array}$ \\
\hline Military $\times$ Protest & $\begin{array}{r}-0.860 \\
(0.88)\end{array}$ & $\begin{array}{l}-1.196 \\
(1.13)\end{array}$ & \\
\hline Military & $\begin{array}{l}1.755 \\
(0.96)\end{array}$ & $\begin{array}{l}2.781^{*} \\
(1.24)\end{array}$ & \\
\hline Military $\times$ Incumbent & $\begin{array}{c}1.671^{* *} \\
(0.59)\end{array}$ & $\begin{array}{l}1.386^{*} \\
(0.70)\end{array}$ & \\
\hline Military $\times$ Coup & $\begin{array}{r}-3.807 \\
(2.41)\end{array}$ & $\begin{array}{c}-5.738^{*} \\
(2.51)\end{array}$ & \\
\hline Coup & $\begin{array}{c}5.274^{* *} \\
(1.49)\end{array}$ & $\begin{array}{c}8.578^{* *} \\
(1.61)\end{array}$ & $\begin{array}{c}-14.493 \\
(16.99)\end{array}$ \\
\hline Prior & $\begin{array}{c}0.820^{* *} \\
(0.30)\end{array}$ & $\begin{array}{c}1.350^{* *} \\
(0.37)\end{array}$ & \\
\hline Incumbent & $\begin{array}{c}0.868^{* *} \\
(0.34)\end{array}$ & $\begin{array}{l}0.781 \\
(0.45)\end{array}$ & \\
\hline Irregular & $\begin{array}{l}0.295 \\
(0.32)\end{array}$ & & $\begin{array}{c}7.901^{* *} \\
(1.79)\end{array}$ \\
\hline IMR & $\begin{array}{c}0.550^{* *} \\
(0.12)\end{array}$ & & $\begin{array}{c}0.693^{* *} \\
(0.19)\end{array}$ \\
\hline Monitor & & $\begin{array}{c}0.855^{*} \\
(0.43)\end{array}$ & \\
\hline Irregular $\times \log ($ duration $)$ & & & $\begin{array}{c}-2.554^{* *} \\
(0.64)\end{array}$ \\
\hline (Intercept) & $\begin{array}{c}-7.976^{* *} \\
(0.77) \\
\end{array}$ & $\begin{array}{c}-8.027^{* *} \\
(0.90)\end{array}$ & $\begin{array}{c}-11.128^{* *} \\
(1.67)\end{array}$ \\
\hline $\ln (\rho)$ & $\begin{array}{c}0.268^{* *} \\
(0.10)\end{array}$ & $\begin{array}{c}0.295^{* *} \\
(0.11)\end{array}$ & $\begin{array}{c}0.740^{* *} \\
(0.18)\end{array}$ \\
\hline Log likelihood & -116.1 & -87.9 & -55.4 \\
\hline Observations & 1423 & 1424 & 1423 \\
\hline
\end{tabular}

